\title{
On the uniqueness of solutions of stochastic differential equations
}

\author{
By \\ Toshio Yamada and Shinzo Watanabe
}

(Received September 21, 1970)

\section{Introduction}

In this paper, we shall discuss the uniqueness problem for solutions of stochastic differential equations.

The theory of stochastic differential equations, as is well known, was developed mainly by Ito and furnishes a very important tool of constructing diffusion processes. Skorohod [4] showed the existence of solutions under the condition that coefficients are only continuous and then, the problem of the uniqueness of solutions becomes important. In order to define a diffusion process through a solution of the stochastic differential equation, it is sufficient to verify the uniqueness in the sense of the probability law of solutions. It may be needless to say that there are many means to verify it; in analytic way, through the theory of differential equations (cf. Stroock-Varadhan [5]) and in probabilistic way through several transformations such as time change or the change of drift.

Here, we shall study mainly the pathwise uniqueness of solutions. In Ito's classical theory where the coefficients are assumed to be Lipschitz continuous, the pathwise uniqueness holds and the solution can be constructed on a given Brownian motion through successive approximation. The uniqueness in the sense of the probability law is obvious in this case. There are several examples where Ito's theory 
can not apply yet we can prove the pathwise uniqueness. Such examples were given by Skorohod [4] and Tanaka [6]. We will improve their results below. As we shall see, the pathwise uniqueness generally implies the uniqueness in the sense of the probability law and thus, the solution defines a unique diffusion process. In this way, we have some examples (besides the Ito's case) of constructing diffusion processes through solutions of stochastic differential equations by verifying the pathwise uniqueness. Other construction of such processes seems to be much more difficult.

Let $\sigma(x)=\left(\sigma_{j}^{i}(x)\right)$ and $b(x)=\left(b^{i}(x)\right), i, j=1,2, \ldots, n$, be defined on $\boldsymbol{R}^{n}$, Borel measurable in $x$ such that $\sigma$ is an $n \times n$-matrix and $b$ is an $n \times 1$-matrix. ${ }^{* 1)} \quad$ We consider the following Ito's stochastic differential equation;

$$
d x_{t}=\sigma\left(x_{t}\right) d B_{t}+b\left(x_{t}\right) d t
$$

or in component wise,

$$
d x_{t}^{i}=\sum_{j=1}^{n} \sigma_{j}^{i}\left(x_{t}\right) d B_{t}^{j}+b^{i}\left(x_{t}\right) d t, \quad i=1,2, \ldots, n
$$

A presice formulation is as follows; by a probability space $(\Omega, \mathscr{F}, P)$ with an increasing family of Borel fields $\mathscr{F}_{t}$, which is denoted as $\left(\Omega, \mathscr{F}, P ; \mathscr{F}_{t}\right)$, we mean a probability space $(\Omega, \mathscr{F}, P)$ with a system $\left\{\mathscr{F}_{t}\right\}_{t \in[0, \infty)}$ of sub-Borel fields of $\mathscr{F}$ such that $\mathscr{F}_{t} \subset \mathscr{F}_{s}$ if $t<s$.

Definition 1. By a solution of the equation (1.1), we mean a probability space with an increasing family of Borel fields $(\Omega, \mathscr{F}, P$; $\left.\mathscr{F}_{t}\right)$ and a family of stochastic processes $\mathfrak{X}=\left\{x_{t}=\left(x_{t}^{1}, x_{t}^{2}, \ldots, x_{t}^{n}\right), B_{t}\right.$ $\left.=\left(B_{t}^{1}, B_{t}^{2}, \ldots, B_{t}^{n}\right)\right\}$ defined on it such that

*1) For the sake of simplicity, we consider the case when the coefficients are independent of $t$ (i.e. temporally homogeneous case) but all the arguments below remain valid when the coefficients are time dependent, i.e. the case when $\sigma=\sigma(t, x)$ and $b=b(t, x)$. 
(i) with probability one, $x_{t}$ and $B_{t}$ are continuous in $t$ and $B_{0}=0$,

(ii) they are adapted to $\mathscr{F}_{t}$, i.e., for each $t, x_{t}$ and $B_{t}$ are $\mathscr{F}_{t^{-}}$measurable,

(iii) $B_{t}$ is a system of $\mathscr{F}_{t}$-martingales such that

$$
\left.<B^{i}, B^{j}>_{t}=\delta_{i j} \bullet, \quad i, j=1,2, \ldots, n, * 2\right)
$$

(iv) $\mathfrak{X}=\left\{x_{t}, B_{t}\right\} \quad$ satisfies

$$
x_{t}-x_{0}=\int_{0}^{t} \sigma\left(x_{s}\right) d B_{s}+\int_{0}^{t} b\left(x_{s}\right) d s
$$

where the integral by $d B_{s}$ is understood in the sense of the stochastic integral.

Remark 1. As is well known (e.g. [3]), $B_{t}$ is an $n$-dimensional Brownian motion such that $B_{t}-B_{s}$ and $\mathscr{F}_{s}$ are independent $(t>s)$.

Now we shall introduce several notions of the uniqueness for solutions of (1.1).

Definitions 2. (Pathwise uniqueness) We shall say that the pathwise uniqueness holds for (1.1) if, for any two solutions $\mathfrak{X}=\left(x_{t}, B_{t}\right)$ and $\mathfrak{X}^{\prime}=\left(x_{t}^{\prime}, B_{t}^{\prime}\right)$ defined on a same probability space $\left(\Omega, \mathscr{F}, P ; \mathscr{F}_{t}\right)$, $x_{0}=x_{0}^{\prime}$ and $B_{t} \equiv B_{t}^{\prime}$ imply $x_{t} \equiv x_{t}^{\prime}$.

When $\sigma$ and $b$ are Lipschitz continuous, then, as is well known by Ito's theory, the pathwise uniqueness holds and $x_{t}$ is constructed on arbitrarily given Brownian motion $B_{t}$ as a measurable function of $x_{0}$ and $B_{t}$.

On the other hand, Skorohod [4] proved the existence of solutions in the sense of Def. 1 for arbitrarily given distribution of $x_{0}$, when $\sigma$

*8) $\left\langle B^{i}, B^{j}\right\rangle_{t}$ is a continuous bounded variation process such that $B_{t}^{i} B_{t}^{j}$ $\left.-<B^{i}, B^{\jmath}\right\rangle_{\iota}$ is an $\mathscr{F}_{t}$-martingale, ([3]). 
and $b$ are only continuous and, in such a general case, the existence and the uniqueness need to be discussed separately.

Definition 3. (Uniqueness in the sense of the probability law) We shall say that the uniqueness in the sense of the probability law holds for (1.1) if, for any two solutions $\mathfrak{X}=\left(x_{t}, B_{t}\right)$ and $\mathfrak{X}^{\prime}=\left(x_{t}^{\prime}, B_{t}^{\prime}\right)^{* 3)}$ such that $x_{0}=x$ and $x_{0}^{\prime}=x$ (a.s.) for some $x \in \boldsymbol{R}^{n}$, the probability law of the processes $x_{t}$ and $x_{t}^{\prime}$ on the space $\{W, \mathscr{B}(W)\}$ coincides, where $W$ is the Fréchet space of all $\boldsymbol{R}^{n}$-valued continuous functions on $[0, \infty)$ with the compact uniform topology and $\mathscr{B}(W)$ is the topological Borel field on $W$.

Stroock-Varadhan [5] proved the existence and the uniqueness in the sense of the probability law of solutions of (1.1) when $\sigma$ is bounded continuous and uniformly elliptic and $b$ is bounded and measurable.

Remark 2. A solution of (1.1) can be defined in a resticted sense as follows; let $Y \subset W$ : by a $Y$-solution of (1.1), we mean a solution $\mathfrak{X}$ of (1.1) such that $P[x .(\omega) \in Y]=1$. The uniqueness (Definitions 2 and 3 ) is defined in the same way and all the propositions and corollaries below remain valid for $Y$-solutions.

Proposition 1. The pathwise uniqueness implies the uniqueness in the sense of the probability law.

Proof. Let $\mathfrak{X}=\left(x_{t}, B_{t}\right)$ and $\mathfrak{X}^{\prime}=\left(x_{t}^{\prime}, B_{t}^{\prime}\right)$ be two solutions of (1.1), (which may be defined on different probability spaces) such that $x_{0}=x$ and $x_{0}^{\prime}=x$ (a.s.). Let $W$ be defined as in Def. 3 and let $P\left(d w_{1} d w_{2}\right)$ and $P^{\prime}\left(d w_{1} d w_{2}\right)$ be the probability law of $\mathfrak{X}$ and $\mathfrak{X}^{\prime}$ on the space $(W \times W, \mathscr{B}(W \times W))$ respectively.

Let $P_{w_{2}}\left(d w_{1}\right)$ be the regular conditional distribution of $P\left(d w_{1} d w_{2}\right)$ given $w_{2}$; i.e., (i) for each $w_{2}$, it is a probability measure on $(\mathbb{W}, \mathscr{B}(W)$ ),

*3) They may be defined on different probability spaces. 
(ii) for each $B \in \mathscr{B}(W), P_{w_{2}}(B)$ is $\mathscr{B}(W)$-measurable in $w_{2}$, (iii) for any $B, B^{\prime} \in \mathscr{B}(W), P\left(B \times B^{\prime}\right)=\int_{B^{\prime}} P_{w_{2}}(B) R\left(d w_{2}\right)$, where $R$ is the probability law of $B_{t}$ on $(W, \mathscr{B}(W))$, i.e., the Wiener measure on $(W, \mathscr{B}(W))$. Similarly $P_{w_{2}}^{\prime}\left(d w_{1}\right)$ is defined for $P^{\prime}\left(d w_{1} d w_{2}\right)$. Define a probability measure $Q\left(d w_{1} d w_{2} d w_{3}\right)$ on $(W \times W \times W, \mathscr{B}(W \times W \times W))$, by

$$
Q\left(d w_{1} d w_{2} d w_{3}\right)=P_{w_{3}}\left(d w_{1}\right) P_{w_{3}}^{\prime}\left(d w_{2}\right) R\left(d w_{3}\right) .
$$

Let $\mathscr{B}_{t}(w)$ be the Borel algebra generated by $w(s), s \leq t$. $\mathscr{B}_{t}(W \times W)$ and $\mathscr{B}_{t}(W \times W \times W)$ are defined similarly. We shall show that $\left[w_{3}(t)\right.$, $\left.\mathscr{B}_{t}(W \times W \times W)\right]$ is a system of martingales with respect to $Q$ such that $\left\langle w_{3}^{i}, w_{3}^{j}\right\rangle_{t}=\delta_{i j} \cdot t$ i.e., $w_{3}$ is an $n$-dimensional Brownian motion such that $w_{3}(t)-w_{3}(s)$ is independent of $\mathscr{B}_{s}(W \times W \times W)$. For this, we need the following

Lemma 1. If $B \in \mathscr{B}_{t}(W), P_{w}(B)\left(P_{w}^{\prime}(B)\right)$ is $\mathscr{B}_{t}(W)$-measurable in $w$.

Proof. Let $P_{w}^{t}(\cdot)$ be the regular conditional distribution given $\mathscr{B}_{t}(W)$; (i) for each $w$, it is a probability measure on $(W, \mathscr{B}(W)$ ), (ii) for each $B \in \mathscr{B}(W), P_{w}^{t}(B)$ is $\mathscr{B}_{t}(W)$-measurable, (iii) for each $B$ $\in \mathscr{B}(W), B^{\prime} \in \mathscr{B}_{t}(W), P\left(B \times B^{\prime}\right)=\int_{B^{\prime}} P_{w}^{t}(B) R(d w)$. It is sufficient to prove that if $B \in \mathscr{B}_{t}(W)$ then $P_{w}(B)=P_{w}^{t}(B)$, a.s.. For this, it is enough to show that, if $F(w)$ is $\mathscr{B}(W)$-measurable and bounded,

$$
\int_{W \times W} F\left(w_{2}\right) I_{B}\left(w_{1}\right) P\left(d w_{1} d w_{2}\right)=\int_{W} F(w) P_{w}^{t}(B) R(d w) .
$$

By the theory of multiple Wiener integrals $([1])$ or by a result in [3], we may assume that

$$
\left.F\left(w_{2}\right)=c+\int_{0}^{\infty} \Phi_{s}\left(w_{2}\right) d w_{2}(s) * 4\right)
$$

*4) $\int_{0}^{\infty} \Phi_{s}\left(w_{2}\right) d w_{2}=\sum_{i=1}^{n} \int_{0}^{\infty} \Phi_{s}^{i}\left(w_{2}\right) d w_{s}^{i}$, and $\Phi_{s}(w)$ is a measurable $\mathscr{B}_{s}(W)$-adapted process. 


$$
=c+\int_{t}^{\infty} \Phi_{s}\left(w_{2}\right) d w_{2}+\int_{0}^{t} \Phi_{s}\left(w_{2}\right) d w_{2} \quad \text { a.a. } w_{2}\left(R\left(d w_{2}\right)\right)
$$

where $c$ is a constant and the integral by $d w_{2}$ is a stochastic integral. Since $\left(B_{t}, \mathscr{F}_{t}\right)$ is a martingale, it is clear that $\left(w_{2}(t), \mathscr{B}_{t}(W \times W)\right.$, $\left.P\left(d w_{1} d w_{2}\right)\right)$ is a martingale. Now,

$$
\begin{aligned}
\int_{W \times W} F\left(w_{2}\right) I_{B}\left(w_{1}\right) P\left(d w_{1} d w_{2}\right) & \\
=c \int_{W \times W} I_{B}\left(w_{1}\right) P\left(d w_{1} d w_{2}\right) & +\int_{W \times W}\left(\int_{t}^{\infty} \Phi_{s}\left(w_{2}\right) d w_{2}\right) I_{B}\left(w_{1}\right) P\left(d w_{1} d w_{2}\right) \\
& +\int_{W \times W}\left(\int_{0}^{t} \Phi_{s}\left(w_{2}\right) d w_{2}\right) I_{B}\left(w_{1}\right) P\left(d w_{1} d w_{2}\right)
\end{aligned}
$$

and the second term is 0 since $\left(w_{2}(t), \mathscr{B}_{t}(W \times W)\right)$ is a martingale and $I_{B}\left(w_{1}\right)$ is $\mathscr{B}_{t}(W \times W)$-measurable. Thus, the above integral is equal to $c \int_{W} P_{w}^{t}(B) R(d w)+\int_{W}\left(\int_{0}^{t} \Phi_{s}(w) d w\right) P_{w}^{t}(B) R(d w)=\int_{W} F(w) P_{w}^{t}(B) R(d w)$.

Now we return to the proof of the proposition. If $F_{1}, F_{2}, F_{3}$ are $\mathscr{B}_{s}(W)$-measurable bounded functions, then

$$
\begin{gathered}
\int_{W \times W \times W}\left[w_{3}^{i}(t)-w w_{3}^{i}(s)\right] F_{1}\left(w_{1}\right) F_{2}\left(w_{2}\right) F_{3}\left(w_{3}\right) Q\left(d w_{1} d w_{2} d w_{3}\right) \\
=\int_{W}\left[w^{i}(t)-w^{i}(s)\right]\left(\int_{W} F_{1}\left(w_{1}\right) P_{w}\left(d w_{1}\right)\right) \\
\quad \times\left(\int_{W} F_{2}\left(w_{2}\right) P_{w}^{\prime}\left(d w_{2}\right)\right) F_{3}(w) R(d w)
\end{gathered}
$$

Since $\int_{W} F_{1}\left(w_{1}\right) P_{w}\left(d w_{1}\right)$ and $\int_{W} F_{2}\left(w_{2}\right) P_{w}^{\prime}\left(d w_{2}\right)$ are $\mathscr{B}_{s}(W)$-measurable in $w$ by the above lemma, the above integral is 0 .

Similarly, we can prove that

$$
\begin{aligned}
& \int_{W \times W \times W}\left\{\left[w_{3}^{i}(t)-w_{3}^{i}(s)\right]\left[w_{3}^{j}(t)-w_{3}^{j}(s)\right]-\delta_{i j}(t-s)\right\} \\
& \times F_{1}\left(w_{1}\right) F_{2}\left(w_{2}\right) F_{3}\left(w_{3}\right) Q\left(d w_{1} d w_{2} d w_{3}\right)=0 .
\end{aligned}
$$


Thus we have proved that $\left[w_{3}(t), \mathscr{B}_{t}(W \times W \times W), Q\right]$ is a system of martingales such that $\left\langle w_{3}^{i}, w_{3}^{j}\right\rangle_{t}=\delta_{i j} \cdot t$. Since $\left(x_{t}, B_{t}\right)$ and $\left(w_{1}, w_{3}\right)$ are the equivalent processes and so are $\left(x_{t}^{\prime}, B_{t}^{\prime}\right)$ and $\left(w_{2}, w_{3}\right)$, we have two solutions $\left(w_{1}, w_{3}\right)$ and $\left(w_{2}, w_{3}\right)$ on the same probability space $\left(W \times W \times W, \mathscr{B}(W \times W \times W), Q ; \mathscr{B}_{t}(W \times W \times W)\right)$. Since $w_{1}(0)=w_{2}(0)$ $=x$ a.s. $(Q)$, the pathwise uniqueness implies $w_{1}(t)=w_{2}(t)$ a.s. $(Q)$. This implies that $P\left(d w_{1} d w_{2}\right)=P^{\prime}\left(d w_{1} d w_{2}\right)$ and hence the uniqueness in the sense of probability law holds. Another consequence is that, for a.a. $w_{3}\left(R\left(d w_{3}\right)\right), P_{w_{3}} \times P_{w_{3}}\left(w_{1}(t)=w_{2}(t)\right)=1$ and this implies that there exists $F(w)$ such that $w_{1}=w_{2}=F\left(w_{3}\right)$. By Lemma 1 , the mapping $w \rightarrow F(w)$ is $\mathscr{B}_{t}(W) / \mathscr{B}_{t}(W)$ measurable. Thus we have

Corollary 1. If the pathwise uniqueness holds and if a solution $\left(x_{t}, B_{t}\right)$ exists such that $x_{0}=x \in \boldsymbol{R}^{n}$, then there exists a function $F(w)$; $w \in W \longrightarrow F(w) \in W$ such that it is $\mathscr{B}_{t}(W) / \mathscr{B}_{t}(W)$-measurable and $x .=F(B$.$) a.s..$

Remark 3. The uniqueness in the sense of probability law does not necessarily imply the pathwise uniqueness. The following example is due to Tanaka. Let $n=1, \sigma(x)=1$ for $x \geqq 0$ and $=-1$ for $x<0$, and $b(x) \equiv 0$. The existence of a solution (1.1) is shown in the following way; let $B(t)$ be a one dimensional Brownian motion and $x(0)$ be a real random variable such that they are mutually independent. Let $x(t)=x(0)+B(t)$ and $\tilde{B}(t)=\int_{0}^{t} \sigma(x(s)) d B(s)$. Then $\tilde{B}(t)$ is a Brownian motion and $[x(t), \tilde{B}(t)]$ is clearly a solution. The uniqueness of the probability law is obvious since, for any solution, $\int_{0}^{t} \sigma(x(s)) d B(s)$ is a Brownian motion. The pathwise uniqueness does not hold since, for $x(0)=0$, if $x(t)$ is a solution then $-x(t)$ is also a solution. Similarly, if $n=1, \sigma(x)=\operatorname{sgn} x|x|^{\beta}, 0<\beta<\frac{1}{2}$ and $b(x) \equiv 0$, and if solutions are restricted to $Y$-solutions where $Y=\left\{w ; \int_{0}^{t} I_{[s ; w(s)=0]} d s=0\right.$, $\left.\mathrm{v}_{t}>0\right\}$, the uniqueness in the sense of probability law holds but the pathwise uniqueness does not hold. 
Proposition 2. Suppose the uniqueness in the sense of the probability law holds. Suppose further that a solution $\left(x_{t}, B_{t}\right)$ of (1.1) exists such that $x_{0}=x$ a.s. for every $x \in \boldsymbol{R}^{n}$ and if $P_{x}$ is the probability law of $x_{t}$ on $(W, \mathscr{B}(W))$ which is unique by the first assumption, $x \rightarrow \rightarrow$ $P_{x}(B)$ is universally measurable for every $B \in \mathscr{B}(W)$. Then $\left\{P_{x}, x \in \boldsymbol{R}^{n}\right\}$ has the strong Markov property.

Proof. This was proved essentially in Stroock-Varadhan [5]. Let $\left(x_{t}, B_{t}\right)$ be a solution on $\left(\Omega, \mathscr{F}, P ; \mathscr{F}_{t}\right)$ and $\sigma$ be an $\mathscr{F}_{t}$-stopping time such that $P(\sigma<\infty)=1$. We assume, as we may, that $\left(x_{t}, B_{t}\right)$ is given as function space-type, i.e., $(\Omega, \mathscr{F})=(W \times W, \mathscr{B}(W \times W))$. Let $P\left(/ \mathscr{F}_{\sigma}\right)$ be the regular conditional distribution given $\mathscr{F}_{\sigma}$. Let $\tilde{x}_{t}=x_{t+\sigma}$, $\tilde{B}_{t}=B_{t+\sigma}-B_{\sigma}$ and $\tilde{\mathscr{F}}_{t}=\mathscr{F}_{t+\sigma}$. Since by Doob's optional sampling theorem,

$$
E\left(B_{t+\sigma}^{i}-B_{s+\sigma}^{i} ; A \cap B\right)=0 \quad \text { for every } A \in \mathscr{F}_{s+\sigma} \text { and } B \in \mathscr{F}_{\sigma} \text {, }
$$

we have $E\left(\tilde{B}_{t}^{i}-\tilde{B}_{s}^{i} ; A / \mathscr{F}_{\sigma}\right)=0$.

Thus, $\left(\tilde{B}_{t}, \tilde{\mathscr{F}}_{t}, P\left(/ \mathscr{F}_{\sigma}\right)\right)$ is a system of martingales. Similarly, we can prove

$$
E\left[\left\{\left(\tilde{B}_{t}^{i}-\tilde{B}_{s}^{i}\right)\left(\tilde{B}_{t}^{j}-\tilde{B}_{s}^{j}\right)-\delta_{i j} \cdot(t-s)\right\} ; A / \mathscr{F}_{\sigma}\right]=0 \quad \mathrm{v} A \in \tilde{\mathscr{F}}_{s} .
$$

Thus, $\left(\tilde{x}_{t}, \tilde{B}_{t}\right)$ is a solution on $\left(\Omega, \mathscr{F}, P\left(/ \mathscr{F}_{\sigma}\right) ; \tilde{F}_{t}\right)$ and the uniqueness in the sense of probability law implies $P\left(\tilde{x} . \in B / F_{\sigma}\right)=P_{x_{\sigma}}(B),{ }^{\vee} B$ $\in \mathscr{B}(W)$.

Corollary 2. Suppose that the uniqueness in the sense of the probability law holds and that, for every Borel probability measure $/ \mathrm{s}$ on $\boldsymbol{R}^{n}$, a solution $\left(x_{t}, B_{t}\right)$ of $(1.1)$ exists such that $P\left[x_{0} \in d x\right]=\mu(d x)$. Then $P_{x}(B), B \in \mathscr{B}(W)$, is universally measurable in $x$ and the probability law $Q$ of $x_{t}$ on $(W, \mathscr{B}(W))$ is given by $Q(B)=\int P_{x}(B) \mu(d x)$, $B \in \mathscr{B}(W)$. Thus $\left\{P_{x}, x \in \boldsymbol{R}^{n}\right\}$ has the strong Markov property. 
Proof. By the proof of Prop. 2, we have

$$
P\left(x . \in B / \mathscr{F}_{0}\right)=P_{x_{0}}(B), \quad{ }^{\vee} B \in \mathscr{B}(W) .
$$

Thus, $P_{x}(B)$ is $\overline{\mathscr{B}}^{\mu}\left(\boldsymbol{R}^{n}\right)^{* 5)}$ measurable and since this holds for every Borel probability measure $\mu, P_{x}(B)$ is $\bigcap_{\mu} \overline{\mathscr{B}}^{\mu}\left(\boldsymbol{R}^{n}\right)$ measurable, that is, universally measurable. Obviously, $Q(B)=P(x . \in B)=\int P_{x}(B) \mu(d x)$.

Corollary 3. Suppose that the pathwise uniqueness holds and that, for every Borel probability measure $\mu$ on $\boldsymbol{R}^{n}$, a solution of (1.1) exists such that $P\left(x_{0} \in d x\right)=\mu(d x)$. Then, there exists a function $F(x, w) ;(x, w) \in \boldsymbol{R}^{n} \times W \longrightarrow F(x, w) \in W$ such that, for every $t$, it is

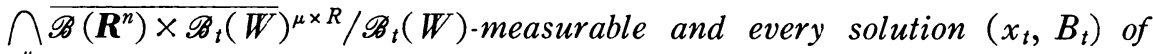
(1.1) satisfies $x .=F\left(x_{0}, B.\right)$ where $R$ is the Wiener measure (i.e., the probability law of $B)$ on $(W, \mathscr{B}(W))$.

Proof. As is proved in Prop. 1, if $x_{0}=x$ a.s., there exists $F(w)$ $\equiv F(x, w): w \cdots F(x, w) \in W$ such that it is $\mathscr{B}_{t}(W) / \mathscr{B}_{t}(W)$-measurable and

$$
x .=F(x, B .), \quad a . s .
$$

Let $\left(x_{t}, B_{t}\right)$ be a solution on $\left(\Omega, \mathscr{F}, P ; \mathscr{F}_{t}\right)$.

Since $\left(x_{t}, B_{t}\right)$ is also a solution on $\left(\Omega, \mathscr{F}, P\left(\cdot / \mathscr{F}_{0}\right) ; \mathscr{F}_{t}\right)$, we have $x .=F\left(x_{0}, B\right)$ a.s. . Now the measurability of $F$ can be proved easily.

Finally, we shall give some non-trivial example of the pathwise uniqueness. Skorohod $([4])$ and Tanaka $([6])$ proved the pathwise uniqueness of the solution, in one-dimensional case, of the equation

$$
d x_{t}=\sigma\left(x_{t}\right) d B_{t}
$$

*5) $\overline{\mathscr{B}}^{\mu}\left(\boldsymbol{R}^{n}\right)$ is the completion of $\mathscr{B}\left(\boldsymbol{R}^{n}\right)$ (=the set of all Borel subsets of $\boldsymbol{R}^{n}$ ) by the measure $\mu$. 
if $\sigma(x)$ satisfies $|\sigma(x)-\sigma(y)| \leqq K|x-y|^{\alpha},{ }^{\mathrm{v}} x, y \in \boldsymbol{R}^{1}$ for some constants $K>0$ and $\alpha>\frac{1}{2}$.

This can be strengthened and the uniqueness holds for $\alpha \geq \frac{1}{2}$.

In fact, we can prove the following

Theorem 1. Let

$$
d x_{t}=\sigma\left(x_{t}\right) d B_{t}+b\left(x_{t}\right) d t,
$$

where

$$
\sigma(x)=\left(\begin{array}{ccc}
\sigma_{1}\left(x_{1}\right) & & 0 \\
& \sigma_{2}\left(x_{2}\right) & \\
& \ddots & \\
0 & & \sigma_{n}\left(x_{n}\right)
\end{array}\right), b(x)=\left(b^{1}(x), b^{2}(x), \ldots, b^{n}(x)\right)^{* 6)}
$$

such that

(i) there exists a positive increasing function $\rho(u), u \in(0, \infty)$ such that

$$
\begin{gathered}
\left|\sigma_{i}(\xi)-\sigma_{i}(\eta)\right| \leq \rho(|\xi-\eta|), \quad \mathrm{v} \xi, \eta \in \boldsymbol{R}^{1}, \quad i=1,2, \ldots, n \\
\text { and } \int_{0+} \rho^{-2}(u) d u=+\infty,
\end{gathered}
$$

(ii) there exists a positive increasing concave function $\kappa(u)$, $u \in(0, \infty)$, such that

$$
\begin{gathered}
\left|b_{i}(x)-b_{i}(y)\right| \leq \kappa(|x-y|), \quad{ }^{\mathrm{v}} x, y \in \boldsymbol{R}^{n}, \quad i=1,2, \ldots, n \\
\text { and } \int_{0_{+}} \kappa^{-1}(u) d u=+\infty .
\end{gathered}
$$

Then the pathwise uniqueness holds for (1.4).

Proof. Let $a_{0}=1>a_{1}>a_{2}>\cdots>a_{k} \rightarrow 0$ be defined by

*b) $x=\left(x_{1}, x_{2}, \cdots, x_{n}\right) \in \boldsymbol{R}^{n}$ 


$$
\int_{a_{1}}^{a_{0}} \rho^{-2}(u) d u=1, \int_{a_{2}}^{a_{1}} \rho^{-2}(u) d u=2, \ldots, \int_{a_{k}}^{a_{k-1}} \rho^{-2}(u) d u=k, \ldots
$$

Then there exists a twice continuously differentiable function $\varphi_{k}(u)$ on $[0, \infty)$ such that $\varphi_{k}(0)=0$,

$$
\varphi_{k}^{\prime}(u)= \begin{cases}0, & 0 \leq u \leq a_{k} \\ \text { between } 0 \text { and } 1, & a_{k}<u<a_{k-1} \\ 1, & u \geq a_{k-1}\end{cases}
$$

and

$$
\varphi_{k}^{*}(u)= \begin{cases}0, & 0 \leq u \leq a_{k} \\ \text { between } 0 \text { and } \frac{2}{k} \rho^{-2}(u), & a_{k}<u<a_{k-1} \\ 0, & u \geq a_{k-1} .\end{cases}
$$

We extend $\varphi_{k}(u)$ on $(-\infty, \infty)$ symmetrically, i.e., $\varphi_{k}(u)=\varphi_{k}(|u|)$. Clearly $\varphi_{k}(u)$ is a twice continuously differentiable function on $(-\infty, \infty)$ such that $\varphi_{k}(u) \uparrow|u|, k \rightarrow \infty$.

Now let $(x(t), B(t))$ and $\left(x^{\prime}(t), B^{\prime}(t)\right)$ be two solutions of (1.4) on the same probability space such that $x(0)=x^{\prime}(0)$ and $B(t) \equiv B^{\prime}(t)$. Then,

$$
\begin{aligned}
x^{i}(t)-x^{\prime i}(t) & =\int_{0}^{t}\left[\sigma_{i}\left(x^{i}(s)\right)-\sigma_{i}\left(x^{\prime i}(s)\right)\right] d B^{i}(s) \\
& +\int_{0}^{t}\left[b_{i}(x(s))-b_{i}\left(x^{\prime}(s)\right)\right] d s, \quad i=1,2, \ldots, n,
\end{aligned}
$$

and by Ito's formula,

$$
\begin{gathered}
\varphi_{k}\left(x^{i}(t)-x^{\prime i}(t)\right)=\int_{0}^{t} \varphi_{k}^{\prime}\left(x^{i}(s)-x^{\prime i}(s)\right)\left[\sigma_{i}\left(x^{i}(s)\right)-\sigma_{i}\left(x^{\prime i}(s)\right)\right] \\
\times d B^{i}(s)+\int_{0}^{t} \varphi_{k}^{\prime}\left(x^{i}(s)-x^{\prime i}(s)\right)\left[b_{i}(x(s))-b_{i}\left(x^{\prime}(s)\right)\right] d s \\
+\frac{1}{2} \int_{0}^{t} \varphi_{k}^{\prime \prime}\left(x^{i}(s)-x^{\prime i}(s)\right)\left[\sigma_{i}\left(x^{i}(s)\right)-\sigma_{i}\left(x^{\prime i}(s)\right)\right]^{2} d s
\end{gathered}
$$




$$
=I_{1}+I_{2}+I_{3}, \text { say. }
$$

Then, $E\left[I_{1}\right]=0$

and, since $\varphi_{k}^{\prime}$ is uniformly bounded,

$$
\left|E\left[I_{2}\right]\right| \leq K_{1} \int_{0}^{t} E\left[\kappa\left(\left|x(s)-x^{\prime}(s)\right|\right)\right] d s \leq K_{1} \int_{0}^{t} \kappa\left(E\left|x(s)-x^{\prime}(s)\right|\right) d s^{* 7)}
$$

by Jensen's inequality.

We have, for $I_{3}$,

$$
\begin{aligned}
\left|I_{3}\right| & \leq \frac{1}{2} \int_{0}^{t} \varphi_{k}^{\prime \prime}\left(x^{i}(s)-x^{\prime i}(s)\right) \rho^{2}\left(\left|x^{i}(s)-x^{\prime i}(s)\right|\right) d s \\
& \leq \frac{1}{2} t_{a_{k} \leq|u| \leq a_{k-1}}\left[\varphi_{k}^{\prime \prime}(u) \rho^{2}(|u|)\right] \leq \frac{1}{2} t \cdot \frac{2}{k} \rightarrow 0, \quad k \rightarrow \infty .
\end{aligned}
$$

Also, $\varphi_{k}\left(x^{i}(t)-x^{\prime i}(t)\right) \uparrow\left|x^{i}(t)-x^{\prime i}(t)\right|$ as $k \rightarrow \infty$. Thus we have

$$
E\left|x^{i}(t)-x^{\prime i}(t)\right| \leq K_{1} \int_{0}^{t} \kappa\left(E\left|x(s)-x^{\prime}(s)\right|\right) d s, \quad i=1,2, \ldots, n
$$

and hence, we have

$$
E\left|x(t)-x^{\prime}(t)\right| \leq K_{2} \int_{0}^{t} \kappa\left(E\left|x(s)-x^{\prime}(s)\right|\right) d s .
$$

As is well known, this implies $E\left|x(t)-x^{\prime}(t)\right| \equiv 0$ and therefore $x(t)$ $\equiv x^{\prime}(t)$. Thus, the pathwise uniqueness holds for solutions of (1.4).

Q.E.D.

As a corollary, the pathwise uniqueness holds for (1.4) if $\sigma$ is Hölder continuous of order $\frac{1}{2}$ and $b$ is Lipschitz continuous.

The condition $\int_{0+} \rho^{-2}(u) d u=\infty$ is, in a certain sense, best possible. For, consider the equation (1.3) when $n=1$. If $\sigma\left(x_{0}\right)=0$ and $\int_{\varepsilon>\left|x-x_{0}\right|>0} \sigma^{-2}(x) d x<\infty$ for some $\varepsilon>0$ there are infinitely many solutions

*7) $K_{1}$ and $K_{2}$ are positive constants. 
of (1.3) just as the example of Girsanov [2].

\author{
Department of Applied Saience \\ FACULTy OF EngineERing \\ KyUShu University \\ Department of Mathematics \\ FACULTY OF Science \\ KYoto UnIVERSITY
}

\title{
References
}

[1] Ito, K., Multiple Wiener Integral, J. Math. Soc. Japan 3 (1951) 157-169.

[2] Girsanov, I. V., An example of non-uniqueness of the solution of the stochastic equation of K. Ito. (English translation) Theory of Prob. and its Appl. 7 (1962) 325-331.

[3] Kunita, H. and S. Watanabe, On square integrable martingales. Nagoya Math. J. 30 (1967), 209-245.

[4] Skorohod, A. V., Studies in the theory of random processes, Addison-Wisley 1965 (Originally published in Kiev)

[5] Stroock, D. W. and S.S. R. Varadhan, Diffusion processes with continuous coefficients, I, II, Comm. Pure Appl. Math. 12 (1969) 345-400 and 479-530

[6] Tanaka, H. and M. Hasegawa, Stochastic differential equations, Vol. 19 of Seminar on Probability (in Japanese) 1964. 